\title{
Survey of knowledge and perceptions of antibiotic resistance and antibiotic stewardship among doctors and pharmacists in a tertiary hospital: a pilot study
}

\author{
Mary Akpan ( m.akpan2@uniuyo.edu.ng ) \\ University of Uyo https://orcid.org/0000-0001-8036-8136 \\ Idong Imo Kingsley \\ University of Uyo \\ Agantem Ekuma \\ University of Uyo \\ Arit Udoh \\ University of Birmingham College of Medical and Dental Sciences
}

\section{Research}

Keywords: Antibiotic resistance, antibiotic stewardship, knowledge and perceptions, doctors, pharmacists, survey

Posted Date: May 7th, 2020

DOl: https://doi.org/10.21203/rs.3.rs-25984/v1

License: (a) This work is licensed under a Creative Commons Attribution 4.0 International License. Read Full License 


\section{Abstract}

Background: Antibiotic resistance poses a serious global public health threat and results in prolonged illness, increased mortality and cost. The WHO global action plan on antibiotic resistance emphasizes five strategic objectives, including improved awareness of antibiotic resistance and optimization of antibiotic use. The objectives of this study were to assess doctors' and pharmacists' knowledge and perceptions of antibiotic resistance and stewardship, and hospital's preparedness to implement antibiotic stewardship programs (ASPs).

Methods: One hundred and twenty-four doctors and 61 pharmacists were surveyed in University of Uyo Teaching Hospital, Akwa Ibom State, Nigeria. Descriptive and Pearson chi-square analysis were carried out to compare knowledge and perceptions between the two groups.

Results: Both doctors and pharmacists perceived antibiotic resistance as a global and national problem (98\% vs $97 \%$ ), respectively. Both groups perceived excessive use of broad-spectrum antibiotics and antibiotics for common cold and cough increase resistance (84\% vs $75 \%, 71 \%$ vs $82 \%$, respectively). Only $47 \%$ of doctors and $53 \%$ of pharmacists correctly defined antibiotic stewardship with $77 \%$ and $85 \%$, respectively, agreeing that restriction of certain antibiotics reduces resistance. There was with no significant difference in knowledge between the two groups in majority of questions/statements; however, there was association between length of practice and knowledge of causes of antibiotic resistance $(F=2.586 ; p=$ 0.028).

Conclusion: Participants in this study showed good knowledge of the prevalence and causes of antibiotic resistance, as well as antibiotic stewardship strategies. There was however little awareness of the hospital's plan to implement ASPs. Hospital management needs to emphasize optimizing antibiotic use through stewardship programs.

\section{Introduction}

Infections caused by antibiotic resistant bacteria are difficult to treat and are associated with increased mortality and increased healthcare cost compared to infections caused by sensitive bacteria [1]. Unnecessary or inappropriate use of antibiotics, such as, antibiotics for nonbacterial infections (influenza, most upper respiratory tract infections (e.g., common cold), yeast infection) or selecting suboptimal type of antibiotic causes the emergence of resistant bacteria [2-5]. The World Health Organization (WHO) surveillance report of resistance in bacteria (E. coli, K. pneumoniae, S. aureus) associated with hospital and community infections showed increased resistance to first line agents [1]. Core objectives of the WHO global action plan on antibiotic resistance include improved awareness and understanding of antibiotic resistance through effective communication, education and training of healthcare professionals and the public, as well as optimization of antibiotics use in human and animal health [6]. The WHO urges all member states to develop national action plan on antibiotic resistance that is aligned with the global action plan [6]. In line with WHO recommendations, the Nigerian government developed the Nigerian National Action Plan on antibiotic resistance 2017-2022 [7]. The objectives of this study were to assess doctors' and pharmacists' knowledge and perceptions of antibiotic resistance and antibiotic stewardship, and hospital's preparedness to implement ASPs.

\section{Methods \\ Study setting and population}

This was a cross-sectional survey of doctors and pharmacists practicing in the University of Uyo Teaching hospital (UUTH). UUTH is a 500-bed hospital located in Uyo metropolis, Akwa Ibom State, South-South Nigeria. It serves a population of about four million people within the state and neighboring states. It records 6000-7000 patient admission/per year. Doctors and pharmacists at any level of practice were eligible to participate in the survey. The doctors were drawn from the different departments of the hospital: Obstetrics and Gynecology, Pediatrics, Chemical Pathology, Internal Medicine, Surgery, Neuropsychiatry, Medical Microbiology, and Anesthesia. Sampling of participants was purposive.

\section{Questionnaire Development}

The questionnaire for this study was developed following review of previous studies [8-12] and national and international reports [7, 8]. The 16-item questionnaire collected information on demographic characteristics of participants, knowledge and perceptions of the prevalence and causes of antibiotic resistance as well as factors that influence prescribing in the hospital. We assessed doctors' and pharmacists' knowledge of antibiotic stewardship by asking them to define antibiotic stewardship and rate their agreement on antibiotic stewardship strategies. To gauge the hospital's preparedness to implement ASPs, we asked questions on the awareness of the national action plan on antibiotic resistance. Statements on prevalence and causes of antibiotic resistance, and antibiotic stewardship strategies were on five-point Likert scale (strongly agree, agree, not sure, strongly disagree, disagree). Questions on the action plan/programs to minimize antibiotic resistance were a combination of 'yes'/'no'/'I don't know' and multiple choice.

We assessed content validity of the instrument by asking antibiotic stewardship expert/antimicrobial resistance strategist from Public Health England to review the questions; feedback was used to improve the instrument. The questionnaire was piloted to ten doctors and pharmacists, respectively to ensure there were no ambiguities. Following the pilot test, questions on hospital plan to implement national action plan/ASPs (Question 16) was modified from five-point scale to 'yes'/'no'/'I don't know' response. The survey instrument is provided as additional file (Additional file 1). 


\section{Data Collection}

We employed paper-based questionnaire which was administered over a six-week period (July 22 to August 26, 2019). Doctors and pharmacists were approached during their clinical or review meetings to complete the questionnaire; completed questionnaires were collected at the close of work on each day. Personal reminders (telephone calls, SMS and office visit) were given after one week for questionnaires that were not completed at close of work on the days meetings were held. Approval for the study was obtained from the University of Uyo Teaching Hospital Institutional Health Research Ethical Committee (UUTH/AD/S/96/VOL.XXI/278). Consent to participate in the survey was sought in the questionnaire.

\section{Data analysis}

Data analysis was done using SSPS software version 25 [IBM SPSS, New York, USA]. Descriptive statistics: frequency and mean were determined. For ease of analysis, knowledge and perception questions on five-point Likert scale were modified to three-point scale. Strongly agree and agree were merged to 'agree' while strongly disagree and disagree were merged to 'disagree'; not sure option was unaltered. For statements on causes of antibiotic resistance and antibiotic stewardship strategies, we assigned scores to participants' responses. 'Agree' response was the correct response and was assigned one point. 'Not sure' and 'disagree' were grouped to be incorrect responses, and were together assigned zero point.

We used Pearson chi-square test to compare knowledge/perceptions of prevalence and causes of antibiotic resistance and strategies to contain antibiotic resistance between the two professional groups (doctors and pharmacists). Furthermore, we sought to determine whether length of practice had any influence on knowledge of causes of antibiotic resistance and antibiotic stewardship strategies between the two groups. We determined sum scores for causes of antibiotic resistance and antibiotic stewardship strategies and used one-way ANOVA to assess influence of length of practice on knowledge. Significant level, p-value was set at $<0.05$

\section{Results}

Of 350 and 79 eligible doctors and pharmacists, respectively, 124 doctors (35\%) and 61 pharmacists (77\%) participated in the survey. Majority of the respondents were males $(110 / 185,60 \%)$, within the age bracket $(25-34$ years). The demographic characteristics of participants are summarized in Table 1. 
Table 1

Demographic characteristics of participants

\begin{tabular}{|c|c|}
\hline Variable & $\begin{array}{l}N=185 \\
n(\%)\end{array}$ \\
\hline \multicolumn{2}{|l|}{ Position } \\
\hline House officers & $34(18)$ \\
\hline Medical officers & $9(5)$ \\
\hline Residents & $62(34)$ \\
\hline Consultants & $19(10)$ \\
\hline Pharmacists & $29(16)$ \\
\hline Intern pharmacists & $32(17)$ \\
\hline \multicolumn{2}{|l|}{ Gender } \\
\hline Male & $110(60)$ \\
\hline Female & $75(40)$ \\
\hline \multicolumn{2}{|c|}{ Length of practice (years) } \\
\hline $1-5$ & $84(45)$ \\
\hline $6-10$ & $47(25)$ \\
\hline $11-15$ & $28(15)$ \\
\hline $16-20$ & $18(10)$ \\
\hline$>20$ & $1(0.5)$ \\
\hline \multicolumn{2}{|l|}{ Age (years) } \\
\hline$<25$ & $19(10)$ \\
\hline $25-34$ & $94(51)$ \\
\hline $35-44$ & $52(28)$ \\
\hline $45-54$ & $17(9)$ \\
\hline $55-64$ & $3(2)$ \\
\hline
\end{tabular}

Insert Table 1 here:

\section{Perception/awareness Of The Burden Of Antibiotic Resistance}

Both doctors and pharmacists perceived antibiotic resistance as a global and national problem ( $98 \%$ vs $97 \%$ ), respectively. There were no significant differences in perception of burden of resistance and/or awareness of the national or hospital rates of antibiotic resistance between doctors and pharmacists, Fig. 1.

Insert Fig. 1 here:

\section{Knowledge/perceptions Of The Causes Of Antibiotic Resistance}

Six statements assessed respondents' knowledge of the causes and/or factors that contribute to spread of antibiotic resistance (Table 2). Majority of the doctors and pharmacists provided the correct response; there was no significant difference in knowledge between the two groups for five of the statements. However, more pharmacists knew that excessive use of antibiotics in agriculture increases antibiotic resistance. 
Knowledge/perceptions about causes of antibiotic resistance among doctors and pharmacists

\begin{tabular}{|c|c|c|c|}
\hline \multirow[t]{2}{*}{ Statements } & \multirow{2}{*}{$\begin{array}{l}\text { Doctors, } n \\
(\%) \\
N=124\end{array}$} & \multirow{2}{*}{$\begin{array}{l}\text { Pharmacists, } n \\
\text { (\%) } \\
\mathrm{N}=61\end{array}$} & \multirow[t]{2}{*}{$\begin{array}{l}\mathrm{p}- \\
\text { value }\end{array}$} \\
\hline & & & \\
\hline Prescribing too many antibiotics causes antibiotic resistance & $\begin{array}{l}101 / 121 \\
(84)\end{array}$ & $48 / 60(80)$ & 0.564 \\
\hline Prescribing antibiotics for coughs, colds and sore throats increases resistance & $\begin{array}{l}87 / 123 \\
(71)\end{array}$ & $49 / 60(82)$ & 0.112 \\
\hline $\begin{array}{l}\text { Prescribing broad-spectrum antibiotics when narrower spectrum alternatives are available increases } \\
\text { antibiotic resistance }\end{array}$ & $\begin{array}{l}104 / 124 \\
(84)\end{array}$ & $45 / 60(75)$ & 0.151 \\
\hline Prescribing antibiotics for too long causes antibiotic resistance & $\begin{array}{l}96 / 121 \\
(79)\end{array}$ & $45 / 60(75)$ & 0.508 \\
\hline Poor hand hygiene contributes to spread of resistant organisms & $\begin{array}{l}97 / 120 \\
(81)\end{array}$ & $49 / 60(82)$ & 0.893 \\
\hline Excessive use of antibiotics in agriculture and livestock causes resistance & $\begin{array}{l}54 / 121 \\
(45)\end{array}$ & $41 / 59(70)$ & 0.002 \\
\hline
\end{tabular}

\section{Factors That Influence Antibiotic Prescribing}

We sought to identify factors that influence doctors' antibiotic prescribing in the study hospital. The main factors that influence antibiotic prescribing were knowledge of antibiotics and infections, microbiology results, evidenced-based guidelines and previous experience (Fig. 2).

Insert Fig. 2 here:

\section{Knowledge Of Programs To Minimize Antibiotic Resistance}

Sixty-four of 124 doctors (52\%) and 38/61 of pharmacists (63\%) were aware of the term 'antimicrobial or antibiotic stewardship'. Only $47 \%$ of doctors and $53 \%$ of pharmacists correctly defined antibiotic stewardship. Majority of doctors and pharmacists provided the correct response to statements on antibiotic stewardship strategies (Table 3). There was no significant difference in knowledge of antibiotic stewardship strategies between doctors and pharmacists, however, more pharmacists $(p=0.013)$ knew that prompt deescalation of empiric antibiotic based on culture minimizes development of antibiotic resistance.

Table 3

Knowledge/perceptions about antibiotic stewardship strategies among doctors and pharmacists

\begin{tabular}{|c|c|c|c|}
\hline \multirow[t]{2}{*}{ Statements } & $\begin{array}{l}\text { Doctors, } n \\
(\%)\end{array}$ & $\begin{array}{l}\text { Pharmacists, } \mathrm{n} \\
(\%)\end{array}$ & \multirow[t]{2}{*}{$\begin{array}{l}\text { p- } \\
\text { value }\end{array}$} \\
\hline & $N=124$ & $N=61$ & \\
\hline Better use of antibiotics will reduce the problem of antibiotic resistance & $\begin{array}{l}122 / 123 \\
(99)\end{array}$ & $59 / 60(98)$ & 0.602 \\
\hline $\begin{array}{l}\text { Using national/local/professional guidelines will contribute to reducing the incidence of antibiotic } \\
\text { resistance }\end{array}$ & $\begin{array}{l}117 / 121 \\
(97)\end{array}$ & $60 / 60(100)$ & 0.154 \\
\hline Restricting use of certain antibiotics or classes of antibiotic will reduce antibiotic resistance & $95 / 123(77)$ & $51 / 60(85)$ & 0.220 \\
\hline Prompt conversion of intravenous to oral antibiotics will reduce antibiotic resistance & $59 / 119(50)$ & $28 / 56(50)$ & 0.956 \\
\hline $\begin{array}{l}\text { Healthcare professionals' education and training on appropriate antibiotic therapy will reduce antibiotic } \\
\text { resistance }\end{array}$ & $\begin{array}{l}122 / 123 \\
(99)\end{array}$ & $59 / 60(98)$ & 0.602 \\
\hline $\begin{array}{l}\text { Prompt de-escalation of empirical antibiotic therapy on the basis of culture results will reduce antibiotic } \\
\text { resistance }\end{array}$ & 95/121 (79) & $55 / 59(93)$ & 0.013 \\
\hline
\end{tabular}

Majority of the doctors and pharmacists (70\% and 72\%, respectively) were not aware of the Nigerian National Action Plan on antibiotic resistance 20172022. Similarly, majority of the doctors and pharmacists responded 'No' or 'I don't know' to statements on their hospital's preparedness to implement antibiotic control measures (Fig. 3).

Insert Fig. 3 here:

\section{Association of length of practice with knowledge of causes of antibiotic resistance and stewardship strategies}


We assessed the influence of length of practice on knowledge of causes of antibiotic resistance and antibioitic stewardship strategies and found that the longer the years of practice, the more knowledgeable participants were of the causes of antibiotic resistance $(F=2.586 ; p=0.028)$. There was no association between length of practice and knowlegdge of antibiotic stewardship strategies $(F=1.185 ; p=0.319)$.

\section{Discussion}

The objectives of this study were to assess doctors' and pharmacists' knowledge and perceptions of antibiotic resistance (prevalence and causes), and antimicrobial stewardship, and hospital's preparedness to implement ASPs. Participants in this study had good knowledge of the burden and causes of antibiotic resistance. Both doctors and pharmacists viewed antibiotic resistance as a global and national problem, as well as a problem in their hospital. Similar findings have been previously reported [8-11]. Although both professional groups perceived practices such as, prescribing antibiotics for common cold and coughs; prescribing broad-spectrum antibiotics when there are suitable narrow-spectrum alternatives and poor hand hygiene contribute to development and/or spread of resistance, less than half of the doctors (45\%) were aware excessive use of antibiotics in agriculture and livestock contributes to resistance burden.

Our findings show that four main factors, namely knowledge of antibiotics and infections, microbiology results, evidenced-based guidelines and previous experience influenced doctors' antibiotic prescribing in the study hospital. These findings are however inconsistent with a previous study to determine the pattern of use of antibiotics in the same facility. In a prospective review of case notes of patients seen at the general outpatient department of the hospital, antibiotics, especially fluoroquinolones were frequently prescribed without microbiology investigations [13]. The authors reported microbiology investigations were requested in only $96 / 511(19 \%)$ of the cases reviewed, with $80 / 96(83 \%)$ of the investigations documented in the case notes and used as a guide for antibiotic prescribing. Findings of a point prevalence survey of antibiotic use in the same facility showed that $97 \%$ of inpatient antibiotic prescribing were empirical; only $3 \%$ were targeted treatment. Futhermore, evidence-based guidelines were not available and/or inaccessble in $95 \%$ of antibiotic indications (Unpublished data). Evidence has shown that doctors' antibiotic prescribing are largely guided by clinical knowledge and experience/expertise rather than guidelines/policies. Even where guidelines are available, they have little influence on antibiotic prescribing, and are somewhat considered obstructive to autonomy and/or subordinate to clinical knowledge and experience [14, 15].

Majority of doctors and pharmacists in this study perceived antibiotic stewardship strategies, such as, use of evidence-based guidelines, education/training and restriction of certain antibiotics are important in reducing development of resistance. However, less than half of the doctors (47\%) and just above average of pharmacists (53\%) correctly defined antibiotic stewardship. This suggests there is little emphasis or awareness on the role of antibiotic stewardship in combating growing antibiotic resistance. In line with WHO recommendations [6], the Nigerian National Action Plan on antibiotic resistance 2017-2022 was published [7]. Interestingly, majority of participants in this study (70\% and $72 \%$, doctors and pharmacists, respectively) were unaware of the existence of such action plan, nor were they aware of their hospital's plan to implement recommendations contained in the action plan. This finding further suggests that while government took the laudable step of developing a national action plan on antibiotic resistance, it exists in the achives of federal ministries. Little or no awareness has been created on its existence or efforts directed at ensuring its implementation. There is therefore a need for government at the national level to give priority to and lead in the fight against antibiotic resistance. This can be achieved through promotion of the national action plan at all levels of healthcare, participation in the World Antibiotic Awareness Week held November each year to create awareness of antibiotic resistance and to encourage best practices among the general public and health workers. The national government can learn from other governments [16-18] and develop and launch antibiotic stwardardship toolkit/guide taking into consideration available resources across all tiers of healthcare in the country.

This study had some limitations. This was a pilot/single-centre study involving only doctors and pharmacists, findings may not be generalized to other settings. A national survey is planned; findings will be compared with those of this pilot study. Secondly, findings presented are self-report of doctors and pharmacists, we could not verify the honesty of participants response.

In conclusion, doctors and pharmacists in this study showed good knowledge of the prevalence and causes of antibiotic resistance, as well as antibiotic stewardship strategies, with no significant difference in knowledge between the two groups in majority of questions/statements. There was however little awareness of the national action plan on antibiotic resistance and hospital's plan to implement recommendations of the national action plan, including institutional antibiotic stewardship programs. Government at all levels and hospital management need to emphasize optimizing antibiotic use through stewardship programs.

\section{Declarations}

\section{Ethics approval and consent to participate}

Ethics approval for this study was sought and obtained from University of Uyo Teaching Hospital Institutional Health Research Ethical Committee (UUTH/AD/S/96/VOL.XXI/278). Consent to participate in the study was sought in the questionnaire; participants were required to check box and consent to participate before proceeding to complete survey.

\section{Consent for publication}

Not applicable 


\section{Availability of data and materials}

The datasets used and/or analysed in this study are available from the corresponding author on reasonable request.

\section{Competing interests}

The authors declare that they have no competing interests

\section{Funding}

This study received no external funding.

\section{Authors' contributions}

Conceptualization, MRA and AE; Data collection, IIK; Analysis, MRA and AU; Writing - Original draft, MRA.; Writing - Review and editing, AE, IIK and AU. All authors read and approved the final manuscript.

\section{Acknowledgements}

The authors would like to thank all doctors and pharmacists who participated in the survey. Dr. Idongesit Jackson of the Department of Clincal Pharmacy \& Biopharmacy, University of Uyo is acknowledged for her review of data analysis and comments on the manuscript prior to submission.

\section{References}

1. World Health Organisation. Antimicrobial Resistance: Global Report on Surveillance. 2014. http://apps.who.int/iris/bitstream/10665/112642/1/9789241564748_eng.pdf?ua=1. Accessed 17 April 2020.

2. Hildreth CJ, Burke AE, Glass RM. Inappropriate Use of Antibiotics JAMA. 2009;302:816.

3. Smieszek T, Pouwels K, Dolk F, Smith FC, Susan Hopkins DR, Sharland S. M et al. Potential for reducing inappropriate antibiotic prescribing in English primary care. J Antimicrob Chemother. 2018;73(Suppl 2):ii36-43. doi:10.1093/jac/dkx500.

4. Spivak ES, Cosgrove SE, Srinivasan A. Measuring appropriate antimicrobial use: attempts at opening the black box. Clin Infect Dis. 2016;63(12):1639-44.

5. Laxminarayan R, Duse A, Wattal C, Zaidi AK, Wertheim HF, Sumpradit N, et al. Antibiotic resistance-the need for global solutions. Lancet Infect Dis. 2013;2:1057-98

6. World Health Organisation. Global action plan on antimicronbial resistance. 2015. https://apps.who.int/iris/bitstream/handle/10665/193736/9789241509763_eng.pdf?sequence=1. Accessed 17 April 2020.

7. Federal Ministries of Agriculture and Rural Development, Environment \& Health. National action plan for antimicrobial resistance 2017-2022. 2017. https://ncdc.gov.ng/themes/common/docs/protocols/77_1511368219.pdf. Accessed 18 April 2020.

8. Pulcini C, Williams F, Molinari N, Davey P, Nathwani D. Junior doctors' knowledge and perceptions of antibiotic resistance and prescribing: a survey in France and Scotland. Clin Microbiol Infect. 2011;17:80-7.

9. Baadani AM, Baig K, Alfahad WA, Aldalbahi S, Omrani AS. Physicians' knowledge, perceptions, and attitudes toward antimicrobial prescribing in Riyadh, Saudi Arabia. Saudi Med J. 2015;36(5):613-9. doi:10.15537/smj.2015.5.11726.

10. Tegagn GT, Yadesa TM, Ahmed Y. Knowledge, attitudes and practices of healthcare professionals towards antimicrobial stewardship and their predictors in Fitche Hospital. J Bioanal Biomed. 2017;9:091-7. doi:10.4172/1948593X.1000159.

11. Labi A, Obeng-Nkrumah N, Bjerrum S, Adu Aryee NA, Ofori-Adjei YA, Yawson AE, Newman MJ. Physicians' knowledge, attitudes, and perceptions concerning antibiotic resistance: a survey in a Ghanaian tertiary care hospital. BMC Health Serv Res. 2018;18:126. doi.org/10.1186/s12913-0182899-y.

12. Waseem H, Ali J, Sarwar F, Khan A, Ur Rehman HS, Choudri M, et al. Assessment of knowledge and attitude trends towards antimicrobial resistance (AMR) among the community members, pharmacists/pharmacy owners and physicians in district Sialkot, Pakistan. Antimicrob Resist Infect Control. 2019;8:67. doi.org/10.1186/s13756-019-0517-3.

13. Eshiet U, Effiong G, Akwaowoh A. The use of antibiotics in a Nigerianh tertiary health care facility. Amer J Biomed Sci Eng. 2015;1(3):25-31.

14. Charani E, Castro-Sánchez E, Sevdalis N, Kyratsis Y, Drumright L, Shah N, Holmes A. Understanding the determinants of antimicrobial prescribing within Hospitals: The role of "prescribing etiquette". Clin Infect Dis. 2013;57:188-96.

15. De Souza V, MacFarlane A, Murphy A, Hanahoe B, Barber A, Cormican M. A qualitative study of factors influencing antimicrobial prescribing by nonconsultant hospital doctors. J Antimicrob Chemother. 2006;58:840-3. 
16. Royal College of General Practitioners. TARGET Antibiotic Toolkit. 2012. https://www.rcgp.org.uk/clinical-andresearch/resources/toolkits/amr/target-antibiotics-toolkit.aspx. Accessed 19 April 2020.

17. Public Health England. Start Smart-Then Focus: Antimicrobial Stewardship Toolkit for English Hospitals. 2015. https://assets.publishing.service.gov.uk/government/uploads/system/uploads/attachment_data/file/417032/Start_Smart_Then_Focus_FINAL.PDF. Accessed 19 April 2020.

18. Department of Health Republic of South Africa. Guidelines on implementation of the antimicrobial strategy in South Africa: One Health Approach \& Governance file:///C:/Users/RICH/Downloads/antimicrobial\%20stewardship\%20guidelines\%20-\%20governance_june2017.pdf. Accessed 19 April 2020.

\section{Figures}

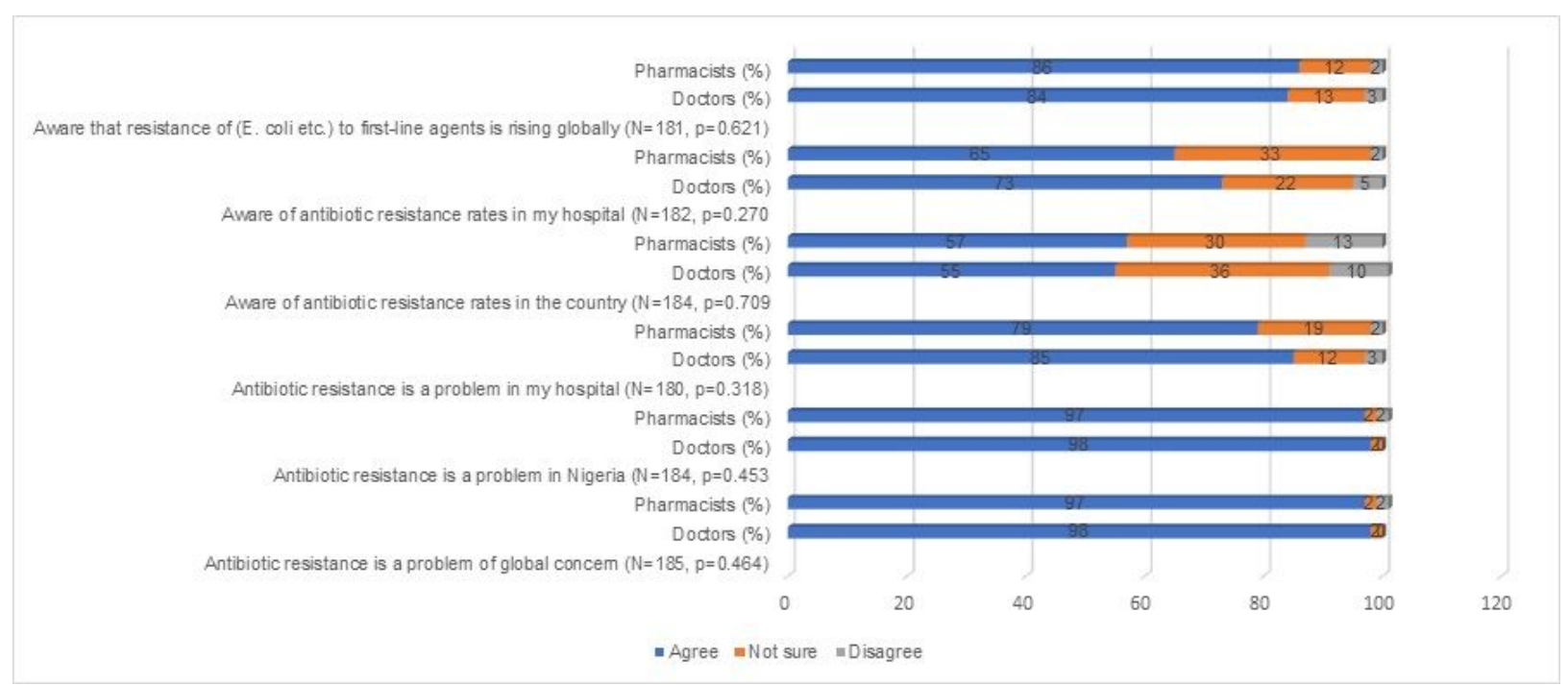

\section{Figure 1}

Perception/awareness of the burden of antibiotic resistance

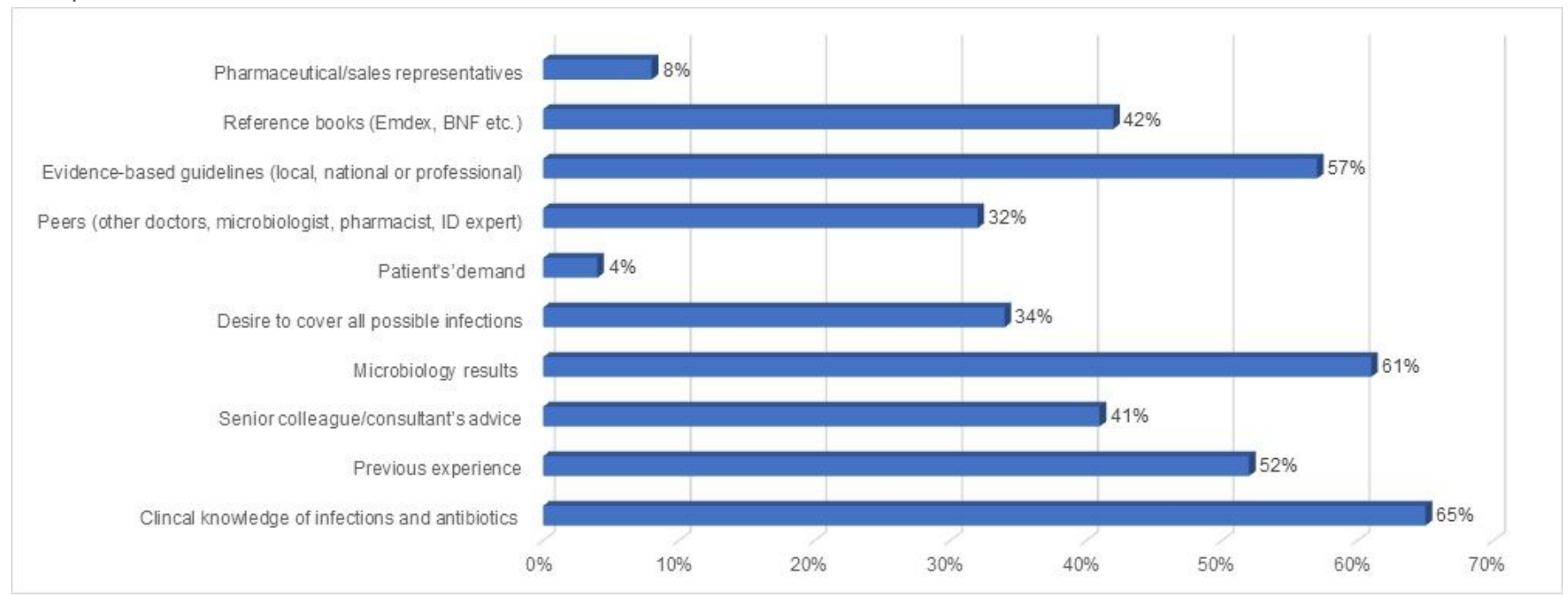

\section{Figure 2}

Factors that influence doctors prescribing. ID - Infectious diseases; BNF- British National Formulary 


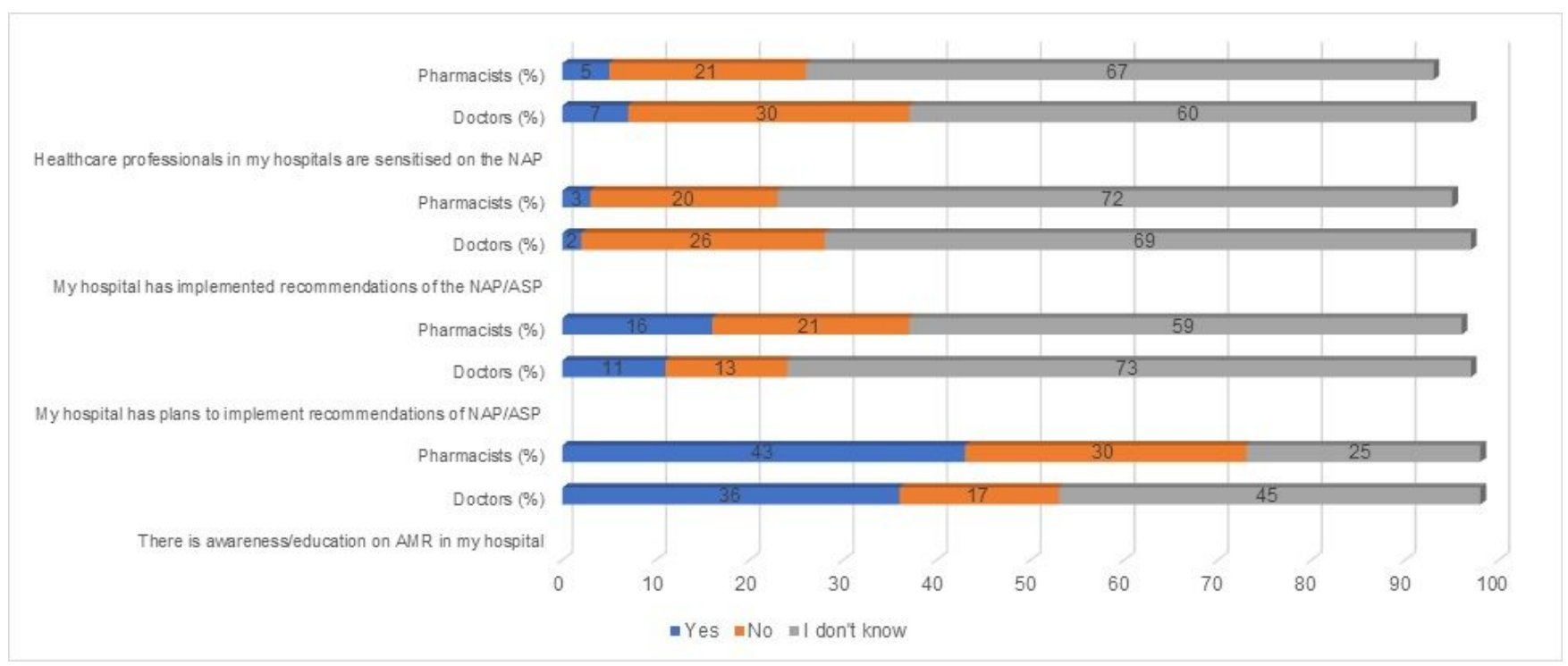

Figure 3

Perception/awareness of hospital's plan to implement recommendations of NAP and stewardship programs. NAP- National Action plan on antibiotic resistance; ASP- antimicrobial/antibiotic stewardship program

\section{Supplementary Files}

This is a list of supplementary files associated with this preprint. Click to download.

- Additionalfile1.docx

- Additionalfile1.docx 